\title{
CARACTERÍSTICAS DE DOCUMENTOS UTILIZADOS PARA ESTRUCTURAR LAS POLÍTICAS INTERNAS RELACIONADAS A LAS METAS INTERNACIONALES DE SEGURIDAD DE PACIENTES ${ }^{1}$
}

\section{CHARACTERISTICS OF DOCUMENTS USED FOR ESTABLISHING INTERNAL POLICIES WHICH AGREE WITH INTERNATIONAL STANDARDS FOR PATIENT SAFETY}

\author{
Ximena Villarroel ${ }^{*}$ \\ NaLdy Febré $\hat{A}^{* *}$
}

\begin{abstract}
RESUMEN
La gestión de riesgos de eventos adversos es un tema fundamental en las instituciones de salud, esta se inicia con la elaboración de normas, protocolos e indicadores, los cuales deben ser construidos de acuerdo a normativas internacionales. Objetivo: Describir las características del documento formal que utilizan las instituciones en nuestro país para estructurar las políticas internas relacionadas a las metas internacionales de seguridad de pacientes. Material y método: Estudio cuantitativo, descriptivo, de corte transversal; siendo evaluados un total de 90 documentos de instituciones de salud pública y privada. Se recolectó la información vía on line, entrevista directa y mediante envío de correos electrónicos a los comités de calidad de las instituciones. Los criterios de selección se basaron en las 6 metas internacionales de seguridad del paciente. Fueron respetadas las normas éticas internacionalmente definidas. Resultados: Se evaluaron un total de 30 instituciones de salud. Los ítems con cumplimiento sobre 90\% fueron "Nombre de la institución y Título del documento". Por su parte "existencia de programa de supervisión " $y$ "presencia de indicador o indicadores" representaron porcentajes de cumplimiento igual a 40\%. De las instituciones evaluadas se encontró la documentación sobre "Identificación de pacientes" en el 90\% (27/30). Conclusión: La presente investigación muestra la diversidad de formatos de estructuración de documentos relacionados a la seguridad de pacientes y exigidos en la acreditación de prestadores.
\end{abstract}

Palabras clave: Seguridad del paciente, normas, investigación en administración de enfermería.

\begin{abstract}
The management of adverse events is a key issue in health institutions; this effort begins with the development of standards, protocols and indicators, which must be built according to international standards. Objective: To assess the official document used by health centers in our country for establishing internal policies with regards to international quality criteria in the area of patient safety. Method: Quantitative, descriptive, cross-sectional study, with a total of 90 documents which were assessed, from private and public health care centers. Information was collected online, through direct interview or e-mails sent to the quality committees of the institutions involved. The selection criteria were based on 6 international patient safety goals. Internationally defined ethical norms were respected. Results: A total of 30 health institutions were assessed. Items reaching above $90 \%$

\footnotetext{
${ }^{1}$ Investigación realizada para la obtención del grado de Magíster en Gestión de Seguridad de pacientes y Calidad de atención, dictado por la Escuela de Enfermería de la Universidad Mayor.

* Matrona Hospital de Carabineros de Chile. Chile. Email: villarroelximena@hotmail.com

** Enfermera. Directora del Programa de Doctorado en Enfermería, Facultad de Enfermería, Universidad Andrés Bello. Chile.Email: nfebre@vtr.net
} 
compliance were "name of institution and title of document". On the other hand, the items "existence of the monitoring program" and "presence of indicator or indicators" had a $40 \%$ compliance. Out of the institutions assessed, documentation about "Patient Identification" was found in $90 \%$ (27/30) of them. Conclusion: The present study shows the diversity of formats for the elaboration of documents for patient safety, which are also required for accreditation.

Key words: Patient safety, standards, nursing administration research.

Fecha recepción: 21/01/14 Fecha aceptación: 16/05/16

\section{INTRODUCCIÓN}

De acuerdo al Marco conceptual de la clasificación internacional para la seguridad del paciente, publicada por la OMS, entenderemos como evento adverso a "todo hecho no deseado que ha causado daño al paciente o lo ha podido causar, ligado tanto a las condiciones de la asistencia como a los del propio paciente y que no se relacionan con el curso natural de la enfermedad" (1).

La Organización Mundial de la Salud (OMS) estima que, a escala mundial, cada año decenas de millones de pacientes sufren lesiones discapacitantes o mueren como consecuencia de prácticas médicas o atención insegura. Uno de cada 10 pacientes sufre algún daño al recibir atención sanitaria en hospitales bien financiados y tecnológicamente adelantados. La atención insegura genera gastos médicos y de hospitalización, infecciones asociadas a la atención de salud, pérdida de ingresos, discapacidad y judicialización llegando a cifras estimadas entre US\$ 6000 millones y US\$ 29.000 millones por año (1).

La seguridad de los pacientes es definida por la Organización Mundial de la Salud (OMS), como "la ausencia de riesgo o daño potencial asociado con la atención sanitaria, que se basa en el conjunto de elementos estructurales, procesos, instrumentos y metodologías basadas en evidencias científicamente probadas con el objeto de minimizar el riesgo de sufrir un evento adverso en el proceso de atención de salud o de mitigar sus consecuencias" (2).

La Joint Commission International (JCI) definió, el año 2005, las 6 Metas Internacionales de Seguridad del Paciente, con el propósito de promover mejoras específicas en cuanto a la seguridad de estos. Destacando las áreas problemáticas dentro de la atención de salud y describiendo soluciones consensuadas para estos problemas, basadas en evidencia científica como en el conocimiento de expertos; las metas internacionales son: 1) identificar correctamente los pacientes; 2) mejorar la comunicación efectiva; 3) mejorar la seguridad de los medicamentos de alto riesgo; 4) garantizar las cirugías en el lugar correcto, con el procedimiento correcto y el paciente correcto;5) reducir el riesgo de infecciones asociadas a la atención en salud (IAAS) y 6) de reducir el riesgo de daño al paciente por causa de caídas (2).

Para medir el rendimiento de las instituciones de salud desde el punto de vista de calidad y seguridad, se han propuesto diversas metodologías; la OMS ha clasificado estos métodos de medición en: auditorías externas, estudios de experiencia de consumo, evaluaciones llevadas a cabo por terceros, índices estadísticos y evaluaciones internas (3).

En Chile, el Ministerio de Salud (MINSAL) ha impulsado un modelo (Acreditación de prestadores Institucionales) que permite el mejoramiento continuo de la calidad y seguridad en los procesos de atención, basados en la reforma de salud del año 2004; este modelo pretende fomentar las decisiones clínicas 
basadas en la mejor evidencia científica y de recursos disponibles. La ley Nacional emitida por el MINSAL Número 20.584 "Que regula los derechos y deberes que tienen las personas en relación a las acciones vinculadas a su atención en salud", en especial en su artículo 4 menciona "toda persona tiene derecho a que, en el marco de la atención de salud que se le brinda, los miembros del equipo de salud y los prestadores institucionales cumplan las normas vigentes en el país, y con los protocolos establecidos, en materia de seguridad de paciente y atención de salud" (4).

La mencionada ley en su artículo cuarto diferencia los documentos en normas y protocolos. Bahadori et al. (5) describen que, según el enfoque del contenido, la metodología de desarrollo y su aplicabilidad, contamos con diversos tipos de documentos, desde el aspecto más general o macro de un problema de salud en particular (Guía Clínica/ Protocolo) hasta el enfoque más específico describiendo instrucciones (procedimiento). En este sentido es importante considerar la definición de la Unidad Técnica de la Intendencia de prestadores, quienes han definido: Norma Técnica: "Documento de ámbito general, contiene actividades obligatorias en relación a la prevención, diagnóstico, tratamiento y rehabilitación de un determinado problema o situación de salud. Es de carácter normativo, generalmente elaborado por la Autoridad Sanitaria o por un grupo de expertos convocados por ella basándose en la evidencia disponible" y Protocolo Clínico: "Documento de ámbito específico-local, definen el cuidado de los pacientes en situaciones y un contexto específico. Generalmente tienen un carácter normativo local. Están basados en el consenso de un grupo de expertos a nivel local e idealmente complementados con la evidencia disponible" (6).

La gestión de riesgo de eventos adversos se inicia en la estructuración de un marco conceptual entregado por la elaboración en cada institución de normativas y protocolos que orienten el actuar del equipo de salud (7). En la estandarización de procesos asistenciales, los diferentes documentos disponibles son denominados de diferentes formas, guías, protocolos, algoritmos. Estas denominaciones no necesariamente responden formalmente al tipo de documento generado, siendo posible que carezcan del rigor metodológico adecuado (8). El Ministerio de Salud chileno, mediante la Subsecretaría de redes asistenciales, establece protocolos y normas sobre seguridad de pacientes y calidad de la atención en salud para ser aplicados por los prestadores institucionales públicos y privados (9). En el mencionado documento se destaca que las normas establecidas sobre seguridad de paciente y calidad de la atención al respecto son: 1) Reporte de eventos adversos y eventos centinelas; 2) Normas de los programas de prevención y control de las infecciones asociadas a la atención de salud; 3 ) Normas de prevención de errores en pacientes sometidos a procesos quirúrgicos; 4) Normas de prevención de errores asociados a transfusiones y 5) Normas de prevención de errores en la atención de salud y de cuidados del paciente. Es importante destacar que independiente del formato utilizado (norma o protocolo) que se adopte para elaborar la documentación, los contenidos deben estar sustentados en la mejor evidencia científica disponible adaptada al contexto local.

La Real Academia Española define como norma: "Regla que se debe seguir o a que se deben ajustar las conductas, tareas, actividades"; desde el punto de vista del Derecho cuando se habla de una norma se habla de un instrumento que regula y existe obligación en su cumplimiento.

La creciente vinculación de lo legal con el sector salud hace necesario diferenciar entre lo que es de cumplimiento obligatorio, de aquello que no lo es y es por ello la importancia del concepto de guía, que es una orientación y no una exigencia como la norma para el derecho, refiriéndose a normas cuando se habla de la organización y funcionamiento 
de los servicios y no en los procesos asistenciales (8).

El manual del estándar general de acreditación para prestadores institucionales de atención cerrada del MINSAL define protocolo como un "documento oficial del prestador, emanado de la jefatura que corresponda, que contiene la descripción de un conjunto ordenado y secuencial de procedimientos o actividades estandarizadas necesarios para realizar con éxito actividades sanitarias específicas". A su vez, este Ministerio en un instructivo establece requisitos básicos para la elaboración de guías clínicas y protocolos, definiendo norma técnica sanitaria como un "documento que impone una o más acciones de carácter obligatorio, cuyo cumplimiento está regulado a través de una resolución exenta emanada del Departamento Jurídico del Ministerio de Salud, una ley u otro instrumento administrativamente válido, y se refiere directamente al manejo promocional, preventivo, diagnóstico, terapéutico o rehabilitador de un problema de salud". Este instructivo define además protocolo como "Instrucciones sobre manejo operativo de problemas de salud determinados, los que serán de carácter referencial. Dichos protocolos sólo serán obligatorios mediante resolución, en caso que exista una causa sanitaria que lo amerite" (9).

Actualmente no se encuentra claramente definido en la literatura médica la diferencia o similitud entre normas y protocolos. Es por esto que la presente investigación pretendió contestar la pregunta: ¿qué tipo de documento formal utilizan las instituciones en nuestro país (Norma o Protocolo) para estructurar las políticas internas relacionadas a: 1) identificación correcta de los pacientes; 2) mejorar la seguridad de los medicamentos de alto riesgo; 3) garantizar las cirugías en el lugar correcto y 4) reducir el riesgo de daño al paciente por causa de caídas?; determinando las características de elaboración de cada documento analizado.
Objetivo: Describir las características del documento formal que utilizan las instituciones en nuestro país para estructurar las políticas internas relacionadas a las metas internacionales de seguridad de pacientes.

\section{MATERIAL Y MÉTODO}

Para lograr el objetivo se diseñó un estudio descriptivo, cuantitativo de corte transversal, seleccionando una muestra intencionada de 90 documentos, durante un período de ocho meses. La unidad de observación estuvo compuesta por los documentos existentes en las clínicas, hospitales del sector público y privado de alta, mediana y baja complejidad (incluye hospitales de las Fuerzas Armadas) e instituciones de salud a nivel primario. De acuerdo a la ubicación geográfica, el 75\% correspondió a la Región Metropolitana, seguido de un 10;5,0;10\%; correspondientes al norte, a la Quinta Región y al sur del país, respectivamente.

Como criterios de inclusión de documentos (Normas y/o Protocolos) se consideró la selección de cuatro de las seis metas internacionales de seguridad de pacientes, definiendo esta selección por la gravedad que pueden producir los eventos adversos y por las exigencias ministeriales que estas políticas se encontraran estructuradas en las diferentes instituciones. Se definió por los autores: a) Identificación correcta de pacientes; b) Garantizar las cirugías en el lugar correcto, con el procedimiento correcto y el paciente correcto; c) Mejorar la seguridad de los medicamentos de alto riesgo; d) Reducir el riesgo de daño al paciente por causa de caídas.

El estudio se diseñó en 3 etapas, en la primera se creó un instrumento para la recolección de datos, estructurada en cuatro partes: parte I: Datos generales de la institución; parte II: Existencia de cada uno de los documentos exigidos (cuatro metas interna- 
cionales de pacientes) y definir la categoría de documento (norma o protocolo); parte III: Estructura del documento, y parte IV: Desarrollo una ficha epidemiológica creada. El instrumento fue validado por 3 expertos con Magíster en calidad asistencial, los cuales realizaron algunas sugerencias. Los investigadores realizaron las correcciones y se realizó una prueba de campo (estudio piloto) en la que fueron seleccionadas 10 normas de instituciones que no tenían relación a las normativas elegidas para esta investigación; se destaca que la ficha presenta un autoinstructivo para facilitar su aplicación y comprensión.

Para la segunda etapa de la presente investigación, la ficha epidemiológica creada, basándose en los criterios ISO para la estructuración de documentos normativos, fue aplicada en 30 centros de salud públicos y privados, de acuerdo a las tres metodologías seleccionadas de recolección: 1) Vía internet: la información fue recolectada en línea a partir de la página principal de cada institución (sitio WEB); 2) entrevista con delegados de calidad y 3 ) envío previo a través de correo electrónico de una carta de invitación a los centros de salud en estudio, solicitando el envío de las normativas elegidas para fines de este estudio a través del mismo correo electrónico.

Luego de la selección, el investigador aplicó la ficha epidemiológica mediante la revisión de la norma o el protocolo recolectado.
Cabe destacar que se mantuvo la confidencialidad de la institución con la omisión del nombre; las normativas evaluadas una vez analizadas fueron eliminadas por los autores.

Para la tercera etapa se realizó el análisis de datos utilizando el sistema SPSS, donde se evaluaron las respuestas precodificadas, lo que permitió su posterior tabulación y análisis estadístico, utilizando análisis descriptivo. Fueron respetadas las normativas éticas internacionalmente establecidas para este tipo de estudio, para lo cual la presente investigación fue aprobada por el Comité de Ética de la Universidad Mayor.

\section{RESULTADOS}

En el periodo en estudio fueron evaluados los documentos de un total de 30 instituciones de salud, las que presentaban variaciones en la existencia de los cuatro documentos solicitados; de acuerdo a esta variación se logró analizar un total de 90 documentos, se destaca que de las 30 instituciones evaluadas el 23,3\% (7/30) se encontraban con una acreditación relacionada a calidad asistencial vigente. Los datos de la Tabla 1 muestran la distribución de frecuencia de las instituciones, donde destaca que el 36,6\% de las instituciones evaluadas pertenecen al ámbito primario.

Tabla 1. Distribución numérica y porcentual según tipo de institución evaluada (n=30), 2013.

\begin{tabular}{lcc}
\hline Tipo de institución & Frecuencia & Porcentaje \\
\hline Pública & 10 & 33,3 \\
Privada & 7 & 23,3 \\
Fuerzas Armadas & 2 & 6,6 \\
Atención primaria en salud & 11 & 36,6 \\
\hline Total & 30 & 100 \\
\hline
\end{tabular}


La distribución numérica y porcentual según tipo de meta internacional de seguridad de pacientes evaluadas se observa en la Tabla 2, donde destaca que, del total de los documentos evaluados, la meta internacional que tiene mayor frecuencia de presentación es Identificación correcta de pacientes con un $32,2 \%$ del total. Se destaca que el $17,7 \%$ corresponde a la meta referente a garantizar las cirugías en el lugar correcto, con el procedimiento correcto y el paciente correcto.

Los datos contenidos en la Tabla 3 muestran que el 56,7\% de los documentos corresponden a normas y un $34,4 \%$ a protocolos, habiendo un $8 \%$ del total de instituciones evaluadas que no contaban con ningún tipo de documentos referentes a las características en estudio.

$\mathrm{Al}$ analizar los datos de los documentos evaluados, de acuerdo a tipo de institución y tipo de documento referente a la meta internacional de pacientes (Tabla 4), podemos observar que en los Hospitales públicos evaluados existió una mayor diversidad de construcción de documentos para las cuatro metas internacionales de seguridad de pacientes evaluadas, destacando que 24 documentos (60\%) fueron abordados como protocolos.

Tabla 2. Distribución numérica y porcentual según tipo de meta internacional de seguridad de pacientes evaluada $(\mathrm{n}=90), 2013$.

\begin{tabular}{lcc}
\hline Nombre del documento & Frecuencia & Porcentaje \\
\hline Identificación correcta de pacientes & 29 & 32,2 \\
$\begin{array}{l}\text { Garantizar cirugías en el lugar correcto, con el procedimiento correcto y } \\
\text { el paciente correcto }\end{array}$ & 16 & 17,7 \\
Seguridad de medicamentos de alto riesgo & 25 & 27,7 \\
Reducción del riesgo de daño al paciente por causa de caídas & 20 & 22,2 \\
\hline Total & 90 & 100 \\
\hline
\end{tabular}

Tabla 3. Distribución numérica y porcentual según tipo de documento de seguridad de pacientes evaluados, $2013(\mathrm{n}=90)$.

\begin{tabular}{lcc}
\hline Tipo de documento & Frecuencia & Porcentaje \\
\hline Norma & 51 & 56,7 \\
Protocolo & 31 & 34,4 \\
Sin documentos & 8 & 8,9 \\
\hline Total & 90 & 100 \\
\hline
\end{tabular}


Tabla 4. Distribución numérica según tipo de institución y tipo de documentación referente a la meta internacional de seguridad de paciente evaluada, 2013 ( $\mathrm{n}=30$ instituciones de salud).

\begin{tabular}{|c|c|c|c|c|c|c|c|c|c|c|c|c|c|}
\hline \multirow{2}{*}{$\begin{array}{l}\text { Meta internacional de seguridad } \\
\text { de paciente evaluada }\end{array}$} & \multicolumn{3}{|c|}{$\begin{array}{l}\text { Hospitales } \\
\text { públicos }\end{array}$} & \multicolumn{3}{|c|}{ Clínicas } & \multicolumn{3}{|c|}{$\begin{array}{c}\text { Hospitales } \\
\text { institucionales }\end{array}$} & \multicolumn{4}{|c|}{$\begin{array}{l}\text { Centros de salud } \\
\text { atención primaria }\end{array}$} \\
\hline & $\mathrm{P}$ & $\mathrm{N}$ & $\mathrm{NE}$ & $\mathrm{P}$ & $\mathrm{N}$ & NE & $\mathrm{P}$ & $\mathrm{N}$ & $\mathrm{NE}$ & $\mathrm{P}$ & $\mathrm{N}$ & $\mathrm{NE}$ & NA \\
\hline Identificación correcta de pacientes & 6 & 2 & 2 & 1 & 6 & 0 & 1 & 1 & 0 & 1 & 9 & 1 & 0 \\
\hline $\begin{array}{l}\text { Garantizar cirugías en el lugar } \\
\text { correcto, con el procedimiento } \\
\text { correcto y el paciente correcto }\end{array}$ & 4 & 3 & 3 & 0 & 6 & 1 & 0 & 1 & 1 & 0 & 0 & 0 & 11 \\
\hline $\begin{array}{l}\text { Seguridad de medicamentos de } \\
\text { alto riesgo }\end{array}$ & 7 & 2 & 1 & 1 & 6 & 0 & 0 & 1 & 1 & 1 & 6 & 4 & 0 \\
\hline $\begin{array}{l}\text { Reducción del riesgo de daño al } \\
\text { paciente por causa de caídas }\end{array}$ & 7 & 2 & 1 & 1 & 6 & 0 & 1 & 0 & 1 & 0 & 0 & 11 & 0 \\
\hline Total & 24 & 9 & 7 & 3 & 24 & 1 & 2 & 3 & 3 & 2 & 15 & 16 & 11 \\
\hline
\end{tabular}

$\mathrm{P}=$ protocolo; $\mathrm{N}=$ Norma; $\mathrm{NE}=$ No Existe en la institución a pesar que es exigido; $\mathrm{NA}=$ No aplica

Un $10,0 \%$ de las instituciones evaluadas no presentaba documentos para identificación correcta de pacientes, seguridad de medicamentos de alto riesgo ni documentación para la reducción del riesgo de caídas. En contrapartida, las Clínicas privadas estructuraron la documentación a nivel de normativas institucional $(85,7 \%)$. Por su parte las instituciones de atención primaria evaluadas presentaron documentación para tres metas internacionales como norma (Identificación correcta de pacientes, Seguridad de medicamentos de alto riesgo y Reducción del riesgo de daño al paciente por causa de caídas), no aplicando la meta relacionada a cirugía segura.

Con respecto a la estructura de los documentos podemos observar que la mayoría de las instituciones evaluadas cuentan en la elaboración de sus normativas con un encabezamiento bien estructurado (91,1\%), cumpliendo en este porcentaje lo referente a nombre de la institución y título de la norma. En el desarrollo del documento llama la atención que en un 41,1\% no se nombra en forma clara la acción alternativa en caso de no poder cumplir la norma. El déficit en la estructuración de los documentos fue encontrado en lo referido a la existencia y elaboración de indicadores, cumpliendo solo en un 23,3\% el ítem existencia de definición de términos, seguido por la existencia de dimensión $(33,3 \%)$ y definición del indicador $(35,6 \%)$.

La distribución de los porcentajes de cumplimiento por ítem en la estructura de los documentos se encuentra demostrada en la Tabla 5. 
Tabla 5: Porcentajes de cumplimientos de los componentes de la estructura del documento.

Estructura del documento

Porcentaje de cumplimiento

1. Encabezamiento

Nombre de la institución

91,1

Título del documento

91,1

Versión (si hubo versiones anteriores)

73,3

Fecha de emisión

Fecha de próxima revisión (mes y año que corresponde actualizarla o revisarla)

73,3

¿El documento se encuentra vigente?

68,9

2. Introduccion (300 caracteres máximo)

3. Existe objetivo del documento

4. Objetivos específicos

Se identifica claramente quién debe aplicar el documento (técnicos/profesionales/administrativos, entre otros)

82

Se identifica el área a la que está asignada (área específica/transversal a toda la institución)

Se identifican responsables de la coordinación/supervisión

Está definido qué motiva el documento (ej.: seguridad de paciente)

Se establecen palabras claves en el documento

¿Existe definición de términos? (pueden ir en anexos)

¿Existen referencias?

\section{Desarrollo del documento}

Se describe claramente los deberes de cada involucrado en el cumplimiento del documento

Se nombra en forma clara la acción alternativa en caso de no poder cumplir la norma o protocolo

Se identifica quien elaboro el documento con:

$\begin{aligned} \text { nombre } & 71,1 \\ \text { cargo } & 72,2 \\ \text { fecha } & 66,7 \\ \text { firma } & 70\end{aligned}$

Se identifica quien revisa el documento con:

$\begin{aligned} \text { nombre } & 82,2 \\ \text { cargo } & 75 \\ \text { fecha } & 75 \\ \text { firma } & 74,4\end{aligned}$

Se identifica quien autoriza el documento con:

\begin{tabular}{|c|c|c|}
\hline & nombre & 82,2 \\
\hline & cargo & 82,2 \\
\hline & fecha & 77,8 \\
\hline & firma & 78,9 \\
\hline Documento autorizado por el director del establecimiento & & 74,4 \\
\hline Existe programa de supervisión & & 38,9 \\
\hline
\end{tabular}


Continuación Tabla 5.

\begin{tabular}{lc}
\hline 6. Indicador para evaluar normativa/protocolo & 35,6 \\
\hline Existe definición del indicador (ej.: calidad de atención) & 48,9 \\
Nombre del indicador (\% de cumplimiento de ...) & 44,4 \\
Tipo de indicador (proceso, resultado, estructura) & 33,3 \\
Dimensión (ej.: seguridad de paciente) & 57,8 \\
Fórmula (ej.: $N^{\circ}$ total de A/ ${ }^{\circ}$ total de A eval x 100) & 35,6 \\
Estándar (óptimo; lo que aspira en condiciones de excelencia técnica) & 57,8 \\
Umbral (meta esperada de adherencia a la norma mayor o = al 85\%) & 23,3 \\
Definición de términos (ej.: referido a norma de riesgo de caída) & 48,9 \\
Fuente de información (Aplicación de programa de ...) & 54,4 \\
Periodicidad (ej.: Mensual x 3 meses en el servicio de ...) & 55,6 \\
Responsable (coordinación/aplicación) & 38,9 \\
Elaboración de informes (ej.: Trimestral)
\end{tabular}

\section{DISCUSIÓN Y CONCLUSIÓN}

La seguridad del paciente es una dimensión clave de la calidad asistencial; los criterios de calidad en la formulación de normas y/o protocolos relacionadas a la seguridad del paciente constituye un aporte importante a las buenas prácticas al interior de las instituciones de salud, facilitando el quehacer para todos quienes participan en el proceso asistencial, permitiendo la difusión de la información a través de criterios uniformes en el cumplimiento de las metas propuestas para estos fines $(6,10)$.

Se analizaron un total de 90 documentos, en lo que se evaluó la operacionalización institucional de las cuatro metas internacionales de seguridad de los pacientes. A base de los resultados presentados, se observó una diferencia en la denominación de los documentos, el $56,6 \%$ de la documentación evaluada correspondía a Normas y el $34,4 \%$ a Protocolos. Se destaca la diferencia de estos datos con los obtenidos por dos autores, quienes mostraron una tasa de adaptación de normas de modelos de excelencia en los hospitales estudiados en rangos de existencia de normativas de un 50 a $70 \%(11,12)$.
Se destaca que la frecuencia del ítem "sin documento" alcanzó cifras de 8,9\%, porcentaje considerado importante por los autores de la presente investigación, pues al no existir ninguna descripción de los procesos evaluados (metas internacionales de seguridad de paciente) permite que estos procesos se tornen inseguros para los pacientes, ya que los sistemas sanitarios requieren que todos los responsables de entregar atención se involucren con las acciones destinadas a mejorar la calidad del servicio en los diversos ámbitos, esto no puede ser logrado sin el conocimiento de los criterios que van a orientar sus acciones, actividad que las instituciones de salud solo logran con documentos de carácter normativo (13).

De acuerdo a esto, el 17,7\% de las instituciones evaluadas posee el documento referente a garantizar las cirugías en el lugar correcto con el procedimiento correcto y el paciente correcto, con mayor frecuencia se estructura como Norma; sin embargo, se destaca que hubo cinco instituciones hospitalarias que NO presentaron este documento, lo que pone en riesgo al paciente quirúrgico, además de no cooperar con el segundo reto mundial seguridad de paciente instaurado por la OMS (2008) "Cirugía segura salva Vi- 
das", representada por la pausa de seguridad (14).

La correcta identificación del paciente es la primera meta internacional de seguridad del paciente, dada su importancia en evitar diferentes eventos adversos tales como: errores de medicación, procedimientos quirúrgicos en pacientes equivocados, entre otros (14, 15). En la presente investigación el $83,3 \%$ posee algún documento referente a identificación de pacientes, estructurado principalmente como Norma.

La normativa tiene una redacción compleja y difícil de entender; lo que genera confusión, incertidumbre jurídica y da pie a criterios de interpretación encontrados y a veces arbitrarios. Al evaluar el marco normativo de las instituciones analizadas, se observa una diferencia de acuerdo al tipo de institución evaluada, donde observamos que las Clínicas en el $87,7 \%$ de los documentos se encontraban en formato de normativa. En cuanto a la estructura del documento, se observa en la misma tabla que los hospitales públicos presentan una mayor elaboración de protocolos $(60 \%)$ en relación a las cuatro metas internacionales evaluadas; esto puede deberse a la recomendación ministerial que señala que estos documentos podrán elaborarse como protocolos en el documento denominado "Elaboración y Gestión de documentos para el Proceso de Acreditación en Salud Recomendaciones para Prestadores Institucionales" $(6,13)$.

Esta diversidad de presentación de documentación se observa en la ciudad de México, donde entre los años 2002 y 2003 se realizó un diagnóstico de las disposiciones normativas, identificándose más de 32 tipos de documentos que regulan la operación y funcionamiento de las instituciones relacionadas a la gestión pública, muchos de ellos iguales en estructura, alcance, contexto de aplicación, pudiendo reducirse este número a 7 ó 10 , demostrándose el exceso de documentos que existen en la regulación de la función públi- ca, tanto en número como en variedad. Las principales conclusiones de los autores fueron: los documentos no alcanzan a cumplir los objetivos para lo que fueron creados produciendo ambigüedad y confusión, dando crédito a criterios de interpretación encontrados, no hay claridad sobre la jerarquía que tiene cada instrumento respecto a los otros, produciendo además una sobrerregulación al interior de las instituciones (16).

De acuerdo a nuestros resultados, a pesar de no haber obtenido una muestra representativa para realizar generalización de los resultados, podemos definir que actualmente en la Región Metropolitana y regiones se elaboran ambos tipos de documentos Normas y Protocolos, diferencia legalmente inexistente pues si ambos son decretados por resolución adquieren el carácter de obligatoriedad.

Desde el punto de vista de acreditación existen Documentos relacionados con la Gestión Clínica, los que describen protocolos y/o procedimientos clínicos con el fin de estandarizar las atenciones de mayor riesgo o críticas para la seguridad del paciente y Documentos relacionados con la Gestión Interna de la Organización: Describen protocolos y/o procedimientos administrativos y/o logísticos orientados a asegurar una adecuada calidad de la atención (6).

Es por lo anterior que, considerando que los protocolos y las normativas desde el punto de vista de la Acreditación de prestadores son sinónimos, los criterios de calidad evaluados en su construcción fueron definidos como similares para términos de esta investigación. Destaca que los ítems que se solicitan en "Encabezamiento" presentaron los porcentajes más elevados de cumplimiento (78\% con rangos de 68,9 a 91\%). Los autores destacan que los ítems mencionados son parte del formato de la estructura del documento por lo tanto es exigible su cumplimiento.

Los ítems que tienen relación al "desarrollo de indicadores", presentaron porcentajes bajos de cumplimiento ( $45 \%$ con rangos de 
23 a 58\%). Al evaluar la literatura esta define que la estructuración de indicadores en salud tiene como principal enfoque la monitorización de la calidad de atención mediante la evaluación del cumplimiento de la Norma o Protocolo institucional establecido, además de comparar instituciones, identificar oportunidades de mejora y evaluar el impacto de las intervenciones $(15,17,18)$.

La monitorización de indicadores en salud debe ser una actividad planificada y sistemática para identificar oportunidades de mejora o situaciones que deben ser estudiadas en profundidad. Es una puerta de entrada a la dinámica de los ciclos de mejora y un componente ineludible de las actividades de diseño o planificación de la calidad (15). La evaluación de la adherencia a las actividades clínicas es actualmente una práctica estándar a nivel hospitalario, siendo que los porcentajes o tasas de adhesión deben ser informados $(17,18)$.

En conclusión: 1) Se observa que las instituciones analizadas presentan dos tipos de documentos frente a las cuatro metas internacionales de seguridad de pacientes analizadas. 2) El 8,9\% de las instituciones evaluadas no presentaban ninguna documentación, lo que pone en riesgo la integridad del paciente. 3) El cumplimiento de los criterios de calidad en la formulación de documentos a nivel de normativas o protocolos se cumple principalmente a nivel de formato. 4) $27 \mathrm{de}$ las 30 instituciones evaluadas cuentan con un documento que rige el proceso de identificación correcta de pacientes. 5) Se destaca que a nivel de hospitales públicos un 12,5\% no presenta documentos institucionales para identificación de pacientes, seguridad de medicamentos de alto riesgo ni de reducción del riesgo de daño al paciente por causa de caídas. 6) El 45\% de los documentos evaluados presentaron mal estructurado el indicador, lo que impide una monitorización sistemática y continua de la calidad de atención.

\section{REFERENCIAS}

1. Organización mundial de la salud (OMS). El marco conceptual de la clasificación internacional para la seguridad del paciente [Internet]. Ginebra: OMS; 2009 [citado 2 jul 2015].p 1-160. Disponible en: http:// www.who.int/patientsafety/-implementation/icps/icps_full_report_es.pdf

2. Joint Commission International. Estándares de acreditación de los hospitales de Joint Commission International. $5^{\mathrm{a}}$ ed. Illinois: Joint Commission International; 2014. $326 \mathrm{p}$.

3. Bahadori H, J Sadeghifar, Hamouzadeh P, Hakimzadeh SM, Nejati M. Combining multiple indicators to assess hospital performance in Iran using the Pabon Lasso Model. Australas Med J. 2011; 4(4): 1759.

4. Ministerio de Salud, Chile. Ley de Derechos y deberes de las personas en relación con acciones vinculadas a su atención en salud. Ley 20.584/2000 [Internet]. Valparaíso: Biblioteca del Congreso Nacional [citado 18 oct 2014]. Disponible en: http://www.leychile.cl/ Navegar?idNorma $=1039348$

5. Bahadori M, Ravangard R, Yaghoubi M, Alimohammadzadeh K. Assessing the service quality of Iran military hospitals: Joint Commission International standards and Analytic Hierarchy Process (AHP) technique. J Educ Health Promot. 2014; 3: 98.

6. Unidad de Asesoría Técnica. Intendencia de Prestadores. Elaboración y Gestión de documentos para el Proceso de Acreditación en Salud: Recomendaciones para Prestadores Institucionales. [Internet]. Santiago de Chile: Superintendencia de Salud; 2013 [citado 15 nov 2013]. 25 p. Disponible en: http://www.supersalud. gob.cl/observatorio/575/articles-8549_ recurso_1.pdf 
7. Chomaly M, Mañalich J, eds. La desconfianza de los impacientes. Reflexiones sobre el cuidado médico y la gestión de riesgos en las instituciones de salud. Santiago de Chile: Mediterráneo; 2006. p 185.

8. Martínez Sagasta C, comp. Estandarizacion de los procesos asistenciales. Calidad en la gestión clínica. Buenos Aires: Ministerio de Salud de la Nación; 2007. 48 p.

9. Ministerio de Salud, República de Chile. División de Planificación y Presupuesto, Departamento de Calidad de Prestadores, Unidad de Evaluación de Tecnologías Sanitarias. Instructivo Establece Requisitos Básicos para la Elaboración de Guías Clínicas y Protocolos del Ministerio de Salud [Internet]. Santiago de Chile: Ministerio de Salud; s.f. [citado 15 nov 2013]. 18 p. Disponible en: http://www.minsal.gob.cl/-portal/url/item/a 1 eab2e3c443d9a8e04001011f017985.pdf

10. Saura Llamas J, Saturno Hernández P. Evaluación y mejora del diseño de los protocolos clínicos. Centro de salud universitario del barrio del Carmen. Murcia. Atención primaria.1994; 13: 355-61.

11. Abbasi S, Tavakoli N, Moslehi M. Readiness of hospitals with quality management systems based on Joint Commission on accreditation standards. Health Inf Manag. 2012; 9: 512.

12. Yousefian S, Harat AT, Fathi M, Ravand M. A proposed adaptation of Joint commission international accreditation standards for hospital-JCI to the health care excellence model. Adv Environ Biol. 2013; 7: 956-67.

13. Superintendencia de Salud. Intendencia de Prestadores. Manual del Estándar general de Acreditación para Prestadores Institucionales de Atención Cerrada
[Internet]. Santiago de Chile: Superintendencia de Salud; 2009 [citado 10 mar 2013]. 50 p. [citado 10 marzo 2013]. Disponible en: http://www.supersalud.gob. cl/observatorio/575/articles-4530_Manual_AC_pdf.pdf

14. Organização Mundial da Saúde (OMS). Segundo desafio global para a segurança do paciente: Cirurgias seguras salvam vidas (orientações para cirurgia segura da OMS). Río de Janeiro: OPAS/MS/ANVISA; 2009. $211 \mathrm{p}$.

15. Agencia de Calidad del Sistema Nacional de Salud Ministerio de Sanidad y Consumo, España. Construcción y validación de indicadores de buenas prácticas sobre seguridad del paciente [Internet]. Madrid: Ministerio de Sanidad y Consumo; 2008 [citado 10 marzo 2015]. 183 p. Disponible en: http://www.msssi.gob.es/ organizacion/sns/planCalidadSNS/docs/ construccionValidacionIndicadoresSeguridadPaciente.pdf

16. Subsecretaría de la Función Pública. Unidad de Políticas de Mejora de la gestión Pública. Guía Para Emitir Documentos Normativos [Internet]. México, D.F.: Secretaría de la Función Pública; 2011 [citado 10 mar 2013]. 49 p. Disponible en: http://www.normateca.sedesol.gob.mx/ work/models/NORMATECA/Normateca/3_Carrousel/8_Guia_doctos/Guia_ doctos_normativos_SFP_230611.pdf

17. Jones C, Gannon B, Wakai A, O'Sullivan $\mathrm{R}$. A systematic review of the cost of data collection for performance monitoring in hospitals. Syst Rev. 2015; 4: 38.

18. Larson E. Monitoring hand higiene: meaningless, harmful, or helpful? Am J Infect Control. 2013, 41(5 Suppl): s42s45. 